\title{
Special features of ketoacidosis episodes of diabetics aged at least 65years in an internal medicine department
}

\begin{abstract}
Objective: The objective of this study was to contribute to a better knowledge of episodes of diabetic ketoacidosis of patients aged 65years and older.

Materials and method: This is a descriptive cross-sectional study that involved 62cases of patients aged 65years and older hospitalized for ketoacidosis in the internal medicine department of Treichville University Hospital and Diabetes Endocrinology Department of Yopougon University Hospital from June 2015 to June 2016.

Results: Obtained are as follows: Epidemiology: Men are the most affected by the disease with a sex ratio of 1.38 and an average age of 68years. Clinic and biology: it is putative type 2 diabetes with $39 \%$ of cases of inaugural ketoacidosis diabetes. Triggers were dominated by infections $(52 \%)$ with malaria at the top of the list. Evolution: regimens on admission were SOS protocol (56.4\%), ICO protocol (44.1\%). The average length of hospital stay was 6 days during which four deaths were recorded. Standardized geriatric assessment: In $82 \%$ of cases patients had a risk of malnutrition, in $84 \%$ of cases patients had a high probability of depression, $85.5 \%$ of our patients were not dependent, they were able to live without aid, and $85.4 \%$ of patients had a strong suspicion of cognitive impairment.
\end{abstract}

Conclusion: Ketoacidosis of patients aged 65years and older is a particular complication of diabetes in senior patients hence the need to push for a geriatric approach for better management.

Keywords: elderly, diabetes, ketoacidosis, standardized geriatric assessment
Volume 4 Issue I - 2017

\author{
Allah Omer Yves Binan,' Absalome Monde, ${ }^{2}$ \\ Nguessan Michel Konan,' Ubrich Venceslas \\ Acko,' Elodie Annemaryse Aidego-Kouassi,' \\ Toussaint Toutou' \\ 'Internal Medicine Department, University Hospital of \\ Treichville, Africa \\ ${ }^{2}$ University of Cocody, Africa
}

Correspondence: Monde Absalome, Medical Biochemistry Laboratory of Abidjan, School of Medical Sciences, BPV 166, University of Cocody, Abidjan, Africa, Tel +225 224805 54, Email monde_abs@yahoo.fr

Received: June 04, 2016 | Published: March 03, 2017

\section{Introduction}

Diabetes mellitus corresponds to a group of metabolic diseases characterized by a fasting blood glucose $\geq 7 \mathrm{mmol} / 1$ or $1.26 \mathrm{~g} / 1$ performed 2 times or a blood glucose $\geq 11 \mathrm{mmol} / 1$ or $2 \mathrm{~g} / 1$ performed at all times of the day. ${ }^{1,2}$ The WHO predicts a worldwide growth of the prevalence of diabetes that is expected to reach 300 million patients in 2025. ${ }^{3}$ Unlike an old opinion considering diabetes mellitus as a disease of rich countries, the disease is becoming a major concern in developing countries, especially those in sub-Saharan Africa. ${ }^{3}$ This phenomenon recognizes several causes including aging of the population, which leads us to be interested particularly in diabetes of the elderly and its complications. Despite the importance of this issue, the literature is poor in descriptive and interventional studies in the elderly of at least 65years. In addition to this "epidemic" progression, the development of diabetes can be punctuated by acute or chronic complications that make it severe. Our work is to describe the clinical and paraclinical features of ketoacidosis and associated co-morbidities of diabetics aged 65years and older in the Ivory Coast.

\section{Materials and methods}

\section{Materials}

Type and context of the study: This was a cross-sectional prospective study with a descriptive purpose that was carried out in the Internal Medicine Department of Treichville University Hospital of and
Diabetes and Endocrinology Department of Yopougon University Hospital between June 2015 and June 2016.

Population study: It involved all patients hospitalized during this period for diabetic ketoacidosis aged 65years and older.

Inclusion criteria: We included in our study all diabetics' men and women aged at least 65 years hospitalized for ketoacidosis who agreed to participate in our study.

\section{Parameters studied}

Data collection means: The collection of data on each patient was performed from his/her hospital record on a standardized individual card. The standardized geriatric assessment of patients was determined using a questionnaire with which we conducted an interview oriented toward, nutritional status, depression, autonomy scale, cognitive disorders.

\section{The parameters studied were}

Epidemiological criteria: Age, gender, occupation, presence or absence of medical coverage.

Diagnostic procedures: For each patient, we specified the type of diabetes; we also specified if decompensation intervened on a known diabetic predisposition or if it was the mode of revelation; we also sought the trigger, performed a comprehensive review and determined blood glucose levels. We assessed glycosuria, acetonuria, 
creatinine, serum potassium and serum sodium. Ketosis was assessed qualitatively by the test strips keto Diastix. Due to our conditions of practice, the determination of ketonemia, determination of blood $\mathrm{pH}$ and alkaline reserve were not always achieved.

Therapeutic methods: SOS Protocol: This is an insulin therapy by auto-macro-pulse pump with rapid insulin. The continuous insulin infusion is done at the rate of $0.10 \mathrm{IU} / \mathrm{kg} / \mathrm{H}$ in hourly monitoring of blood glucose. When it drops below $2.5 \mathrm{~g} / 1$, the dose of insulin is reduced by half. O.C.I. Protocol: The O.C.I. or optimized conventional insulin therapy consists in the administration of rapid insulin subcutaneously, according to a scheme with four injections as shown in the figure below.

\section{Standardized geriatric assessment}

Functional status: ${ }^{4}$ Its assessment is one of the most important steps. Capacities for basic activities of daily living are measured by the ADL scale (Activities of Daily Living). More complex activities of everyday life are assessed by the IADL (Instrumental Activities of Daily Living).

Cognitive assessment: ${ }^{4}$ Cognitive impairment can have different causes (iatrogenic, metabolic ...), but Alzheimer's disease is the most common cause. Its prevalence increases with age $(5 \%$ among patients over 65years and 30\% among patients over 85years). Cognitive assessment allows early detection of disorders. In our study we used the SCORE of DAKAR which assesses in ten minutes the different aspects of cognitive functions (orientation in time and space, learning, attention and calculation, short-term memory, language, praxis).

Depression: ${ }^{4}$ Various tools have been developed, including the geriatric depression scale (Geriatric Depression Scale, or GDS). If screening is positive, the patient should benefit from a review, an accurate diagnosis and a possible treatment.

Nutrition: ${ }^{2}$ The Mini Nutritional Assessment, or MNA, is a validated and standardized stool assessing nutritional status. In addition to its diagnostic value, it helps carry out a small nutritional survey to investigate the possible causes contributing to malnutrition and therefore guide the means of intervention.

\section{Results}

\section{Age (Figure I)}

\section{Age}

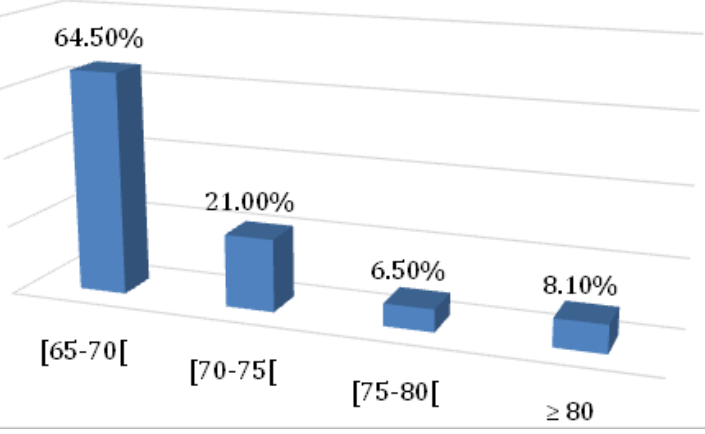

Figure I Distribution of cases of keto acidosis according to age groups.

\section{Precipitating factors (Table I)}

Table I Distribution of cases of ketoacidosis as the trigger

\begin{tabular}{lcc}
\hline Cause of decompensation & Number & Percentage \\
\hline Stop treatment & 4 & $6 \%$ \\
Inaugural & 24 & $39 \%$ \\
Infections & 32 & $52 \%$ \\
Not found & 2 & $3 \%$ \\
& & \\
Total & 62 & $100 \%$ \\
\hline
\end{tabular}

In $52 \%$ of cases the trigger was infectious

\section{Glucose at admission (Figure 2)}

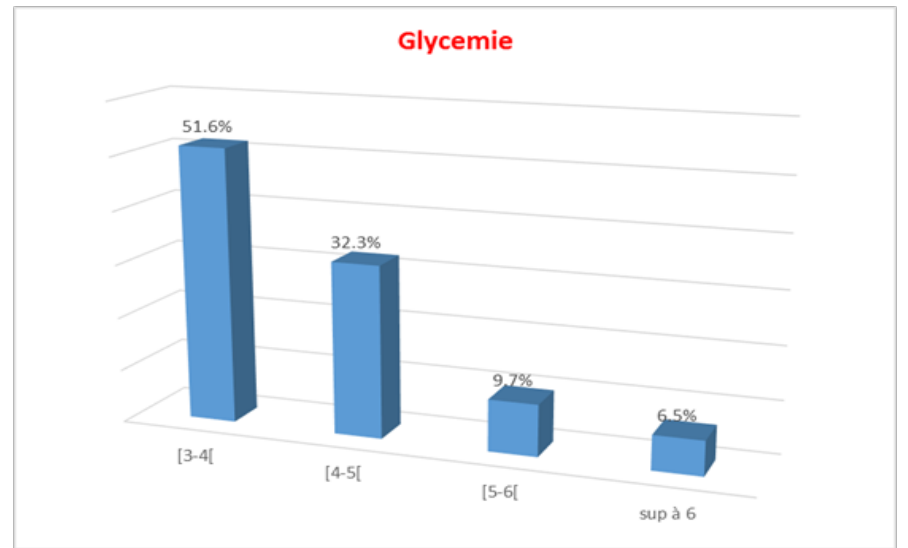

Figure 2 Distribution of cases of keto acidosis according to the input blood sugar.

\section{Glycosuria (Figure 3)}

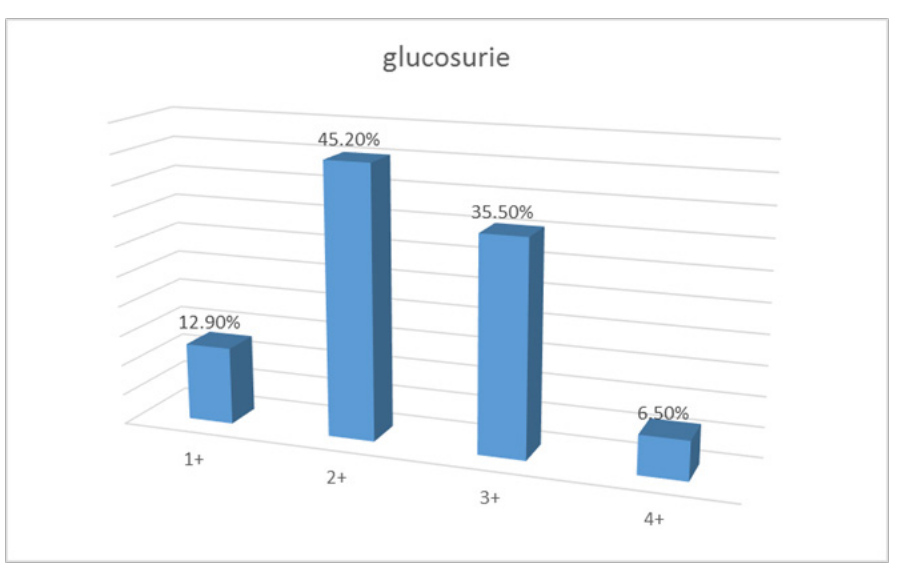

Figure 3 Distribution of cases of keto acidosis according glycosuria. 


\section{Acetonuria (Figure 4) (Table 2)}

Table 2 Distribution of cases of keto acidosis by blood diagnostic tests and their results

\begin{tabular}{lll}
\hline Review paraclinique & Number & Percentage \\
\hline Blood sugar & 62 & $100 \%$ \\
High blood sugar & 62 & $100 \%$ \\
Natremia & 62 & $100 \%$ \\
Hyponatrémia $(\leq 135 \mathrm{meq} / \mathrm{l})$ & 21 & $33.90 \%$ \\
NORMAL $(135-145 \mathrm{meq} / \mathrm{l})$ & 33 & $53.20 \%$ \\
Hypernatrémie $(\geq 145 \mathrm{meq} / \mathrm{l})$ & 8 & $12.90 \%$ \\
Kaliemie & 62 & $100 \%$ \\
Hypokaliémia $(\leq 3,5 \mathrm{meq} / \mathrm{l})$ & 6 & $9.70 \%$ \\
Normal $(3,5-5 \mathrm{meq} / \mathrm{l})$ & 48 & $77.40 \%$ \\
Hyperkaliémia $(\geq 5 \mathrm{meq} / \mathrm{l})$ & 8 & $12.90 \%$ \\
\hline
\end{tabular}

We found hyponatremia in $33.9 \%$ of cases and hypokalemia in $9.7 \%$ of cases

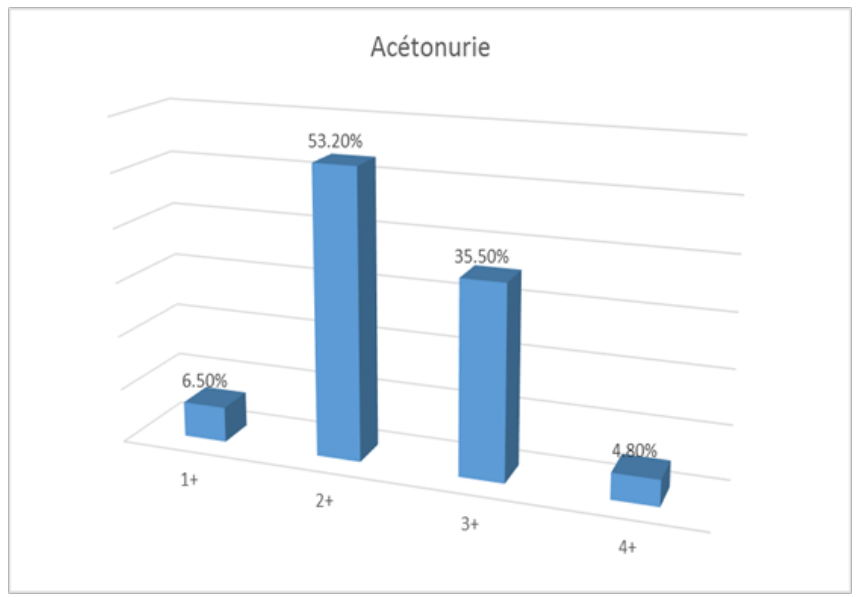

Figure 4 Distribution of cases of keto acidosis according acétonuria

\section{Geriatric standardized assessment}

Mini nutritional assessment (MNA) (Figure 5)

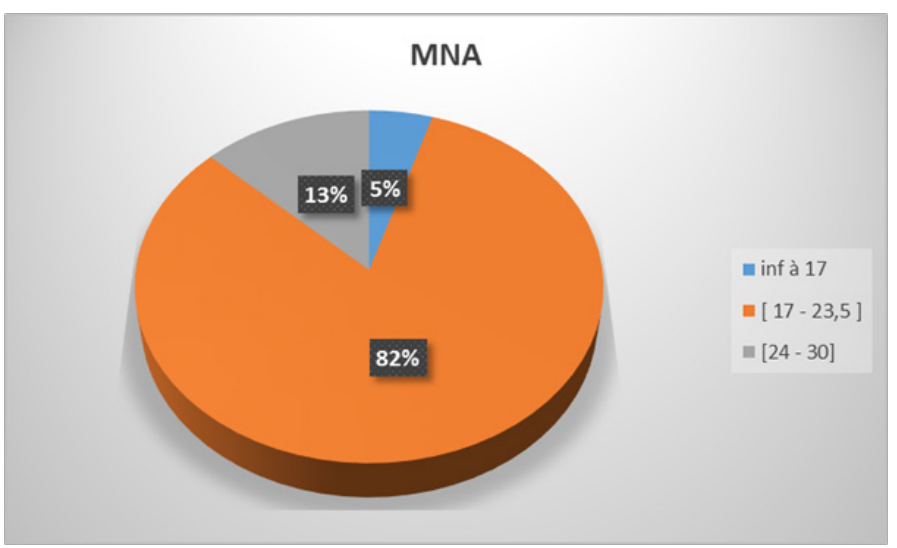

Figure 5 Distribution of patients according to the nutritional status.
Mini gériatric dépression scale (Figure 6)

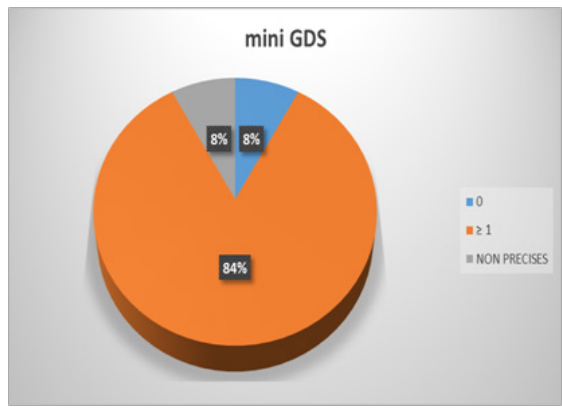

Figure 6 Distribution of patients according to depression.

Score to use the tools of daily living (IADL scale) (Figure 7)

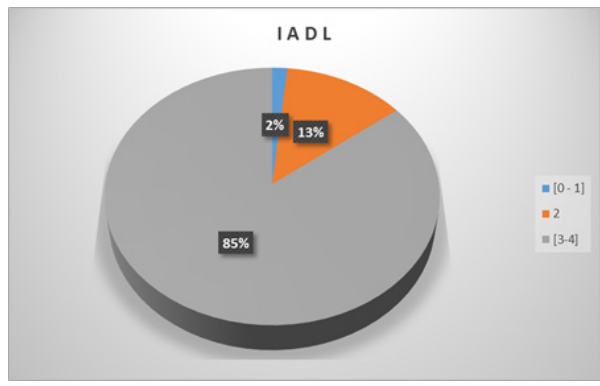

Figure 7 Distribution of patients according to the scale IADL.

\section{Autonomy scale ADL (Figure 8)}

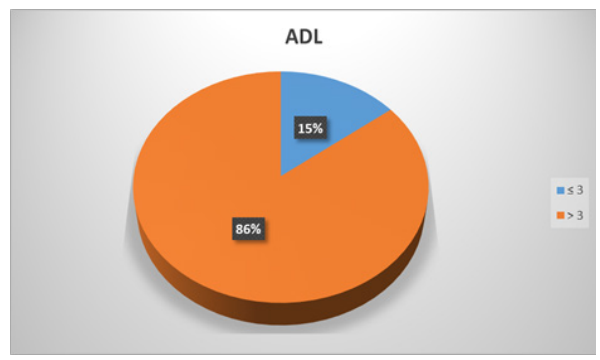

Figure 8 Cases distribution according ADL's scale.

\section{Sénégal test (Figure 9) (Table 3) (Table 4)}

Table 3 Cases of keto acidosis distribution according the sexe and the depression

\begin{tabular}{lllll}
\hline Mini GDS & Masculin & Percentage & Féminin & Percentage \\
\hline 0 & 3 & $9 \%$ & 2 & $8 \%$ \\
$\geq 1$ & 30 & $91 \%$ & 22 & $92 \%$ \\
Total & 33 & $100 \%$ & 24 & $100 \%$ \\
\hline
\end{tabular}

Odds ratio I.I, p 0.65 , no significative

Table 4 Ketoacidocis deaths distribution according the sex

\begin{tabular}{lllll}
\hline Deaths & Masculin & Percentage & Féminin & Percentage \\
\hline Oui & 3 & $8 \%$ & 1 & $4 \%$ \\
Non & 33 & $92 \%$ & 25 & $96 \%$ \\
Total & 36 & $100 \%$ & 26 & $100 \%$ \\
\hline
\end{tabular}

Odds ratio 2.27; p 0.43; no significative 


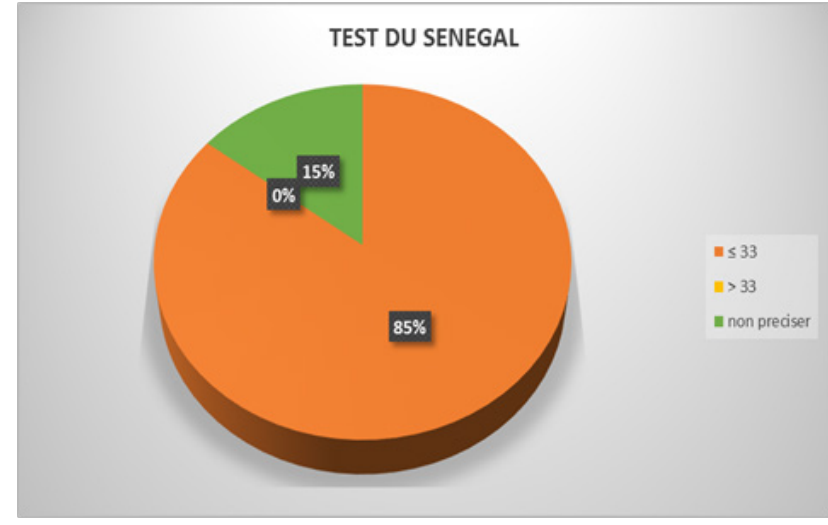

Figure 9 Cases distribution according Senegal Test.

\section{Discussion}

\section{Epidemiology}

At the end of our study we recorded 62 patients over a period of 12 months. The prevalence of ketoacidosis of patients over 65 years has not been established because our study was a multicenter one but $\mathrm{Abodo}^{5}$ found in his study a prevalence of $20 \%$. The average age was 68years with a maximum of $85 y$ years and a minimum of 66years, a significant proportion ( $85 \%)$ between [65 and 74]. These results are similar to those of $\mathrm{Alix}^{6}$ and $\mathrm{Abodo}^{5}$ who found an average age of 68years and 71.8years respectively. We also noted a male predominance (sex ratio 1.38 ) and type 2 diabetes in all our cases. This could be explained by the fact that type 2 diabetes is the most common in the world and it involves in general adult subjects. Our results corroborate those of $\mathrm{Alix}^{6}$ who found a male predominance. Abodo $^{5}$ found no difference between men and women. Thus none of them benefited from social security, which raised a real problem of management of diabetes in the elderly since diabetes is a chronic condition. Hypertension was found in $38.7 \%$ of cases. Indeed, the Hypertension/Diabetes combination is common and dreaded because of their cumulative comorbidity. Abodo ${ }^{5}$ also found the same association in $46.65 \%$ of cases. Smoking has been found meanwhile in $30.6 \%$ of cases and alcoholism in $19.4 \%$ of cases.

\section{Clinical examination}

In our study, the ketoacidosis of the elderly was inaugural in $39 \%$ of cases of type 2 diabetes whereas ketoacidosis is a complication of typeldiabetes and therefore little common in the elderly. ${ }^{7}$ It may sometimes occur in a type 2 diabetic patient of with a long duration of evolution and who has become very insulin deficient, due to the decrease in insulin secretion, in the kinetics of slower insulin secretion, the decrease in physical activity and an increase in fat mass. ${ }^{8}{ }^{\text {Alix }}{ }^{6}$ found inaugural diabetes in half of the cases while $\mathrm{Abodo}^{5}$ found $21.22 \%$. The ketoacidosis of the elderly was diagnosed in $92 \%$ of cases in the phase of ketoacidosis pre-coma against $8 \%$ of cases for advanced comas. This high rate of ketosis without acidosis found could be explained by the systematic hospitalization of patients who presented traces of ketonuria without impaired consciousness, for the sake of early management conditioning the subsequent prognosis of ketoacidosis. The dominant symptoms are polyuria $(100 \%)$ of cases, polydipsia and thirst $93.5 \%$ each. These symptoms are also found in Remington. ${ }^{9}$ The mechanism being altered during aging, we need a thorough clinical examination especially the dryness of the oral mucosa and hypotonia of eyeballs, which suggest thirst. ${ }^{9}$ In $84 \%$ of cases, disorders of consciousness were dominated by confusion. Bourdel-Marchasson $\mathrm{J}^{7}$ found stupor in a variable confused state and dehydration.

\section{Biology}

Glycosuria was massive (three or four crosses) in $41 \%$ of cases and acetonuria in $39 \%$ of cases. The average blood glucose of patients on admission was $389 \mathrm{~g} / 1$ with $51.6 \%$ between $[3 \mathrm{~g} / \mathrm{l}-4 \mathrm{~g} / 1]$ a maximum of $6 \mathrm{~g} / \mathrm{l}$ and a minimum of $3 \mathrm{~g} / \mathrm{l}$. Hypokalemia and hyponatremia were found in respectively $9.7 \%$ and $33.9 \%$ of cases. Hyponatremia is related to extracellular dehydration during which there is a rise in urinary sodium $\geq 30 \mathrm{mmol} / 24 \mathrm{~h} .{ }^{10}$ Alix ${ }^{6}$ found a massive glycosuria in $22 \%$ of cases and a massive acetonuria in only $10 \%$ of cases. The average blood glucose of patients on admission was $3.89 \mathrm{~g} / 1$. The determination of $\mathrm{PH}$, the determination of alkaline reserve and Ketonemia as well as glycated hemoglobin have not been achieved and have not been taken into account.

\section{Etiology}

The cause explaining the occurrence of ketoacidosis was found in almost all cases. However among the causes implicated here, as elsewhere in Africa, infection was the most common triggering factor. ${ }^{11-13}$ We found it in $52 \%$ of cases dominated by malaria confirmed by a positive thick blood smear or by a positive antimalarial treatment test in $46.8 \%$ of cases. So we recommend that any malaria attack in a known diabetic elderly over 65years must lead to increase clinical and blood glucose monitoring, or even hospitalization. These results are similar to those of Alix ${ }^{6}$ who found infection as the cause of decompensation in $50 \%$ of cases. Among infectious causes we have acute pyelonephritis, cystitis, tooth abscess. The diabetic elderly is particularly vulnerable vis-à-vis infections. The infection takes hold and develops more easily in a context of frank hyperglycemia and helps accentuate the glycemic control. ${ }^{8}$

\section{Therapy}

At present, treatment of ketoacidosis in its point concerning "restoring the metabolic balance" (insulin therapy), is based on the use of the insulin macro-pump, whose regular rate and capacity to administer continuously low doses of insulin helps avoid the main problem of treatment which is hypoglycemia, which may constitute the dreaded and fatal source of cerebral edema. A hydroelectric rehydration was performed in all patients, in the way to bring 6liters to 8liters within the first eighteen hours (half of which in the first six hours) as shown in the literature. ${ }^{14}$ Potassium intake was early performed from the third hour, in the absence of anuria. Alkalization based on the observation of clinical signs particularly polypnea of Kussmaul in the absence of the determination of alkaline reserves, was performed with caution (50 to $750 \mathrm{CC}$ maximum of bicarbonate in serum $14 \%$ in one hour $)^{15}$ and involved only $1.8 \%$ of patients due to the impossibility to determine the alkaline reserve. This caution in the administration of bicarbonate during the treatment of ketoacidosis is suggested by several authors. ${ }^{11}$ The treatment of the triggering cause, mainly of infectious nature $(51.6 \%)$, by empirical antibiotic therapy or an anti-malaria treatment was systematically associated when it became necessary. In all patients a $<<$ careful monitoring $>>$ was instituted. Let's note that in Africa, Lokrou ${ }^{16}$ with a stopgap protocol of bolus insulin therapy that was performed by acetone cross and in the presence of glycosuria has found the same effectiveness and the same safety with one or the other of protocols. This stopgap protocol was achieved in $38.71 \%$ of cases. 


\section{Outcome}

We recorded 4 deaths $(6.45 \%)$. The picture of ketoacidosis coma appears less serious and the outcome very satisfactory when the management is rapid; it is therefore from the precocity of treatment that depends primarily the patient's survival. ${ }^{17}$ Alix ${ }^{6}$ found a positive outcome in $100 \%$ of cases. The analysis of causes of death showed a patient dead from stroke and another from chronic renal failure CRF. In $3.22 \%$ of cases no cause was found. Severe hypokalemia that was most often related to treatment involved only one patient. Indeed, its occurrence was minimized by a low-dose insulin therapy by continuous infusion. During hospitalization, $8.1 \%$ of patients presented moderate iatrogenic hypoglycemia without coma.

\section{Standardised geriatric assessment}

The nutritional status of patients assessed by the Mini Nutritional Assessment allowed to note that in $82 \%$ of cases patients had a risk of malnutrition and $5 \%$ had poor nutritional status. These results were found by Vischer. ${ }^{18}$ Depression assessed by the Mini Geriatric Depression Scale noted that in $84 \%$ of cases patients had a high probability of depression. This could be explained by the fact that our study population consists mainly of people without full time professional activity. Currently there is a renewed interest in this association, whatever the age of the study population and the type of diabetes. The prevalence of depression is higher among diabetics than non-diabetics, reaching in some studies more than $30 \%{ }^{19}$

In the French survey paquid, carried out among ambulatory patients over 65years, $21.3 \%$ of diabetics are depressed against $12.7 \%$ of non-diabetics. ${ }^{20}$ Although the temporal relationship is difficult to specify the presence of depression in diabetics over 65years is associated with a significant increase in rates of ophthalmologic and macro-angiopathic complications of diabetes (Myocardial Infarction, Stroke, Angor) but also of the incidence of cancer, and osteoarthritis. Functionally, the diabetes-depression association is a dependence risk factor for activities of daily living (walking, bathing, dressing, eating, etc. ...) and instrumental activities (budget management, use of telephone, shopping, etc ....). ${ }^{21}$ Depression among diabetic elderly is a powerful predictor of hospitalization and death. ${ }^{22}$ In depressed patients, glycemic control is less good, the glycated hemoglobin being higher than $1 \%$ on average. ${ }^{23}$ In all elderly, we must always ask the question about the existence of a possible associated depression and manage it, especially faced with an unexplained imbalance of diabetes. Regarding the use of tools of everyday living, assessed by the Instrumental Activity of Daily living Scale, $85.5 \%$ of our patients were not dependent. They were able to live without any help. The assessment of the ability to perform activities of daily living was assessed by the Activity of Daily Living Scale. Let's note that only $14.5 \%$ of our patients had a significant dependence. As for The SENEGAL test, it helps detect that $85.4 \%$ of patients had a strong suspicion of cognitive impairment. $14.52 \%$ of our patients did not meet the conditions for carrying out the test. In descriptive crosssectional studies, diabetic elderly have poorer cognitive performance than non-diabetics of the same age, and among diabetics, the degree of cognitive impairment is correlated with the quality of glycemic control. ${ }^{24}$ Some experimental data emphasize the deleterious effect of chronic hyperglycemia on amnesic process, but there are other factors as hyperinsulinism, hypertension, hypertriglyceridemia. ${ }^{25}$ This risk of cognitive impairment was specified during the follow up of a cohort of women aged from 65 to 99years for 6years: in diabetics, the performance is worse at baseline, and cognitive decline over 6 years is more common and more important than in non diabetics especially for a duration of evolution of diabetes of more than 15 years. ${ }^{25}$ Does improvement in glycemic control prevents cognitive decline? The literature does not provide sufficient evidence to answer this question. Two relatively old studies show improved performance, mainly amnesic and verbal, with a decrease in blood glucose and glycated hemoglobin over 6months, but this involved a small number of patients (16 and 30$){ }^{26,27}$

\section{Conclusion}

Diabetes is a major public health problem given the rapid growth of diabetes worldwide. Acute complications including ketoacidosis are still worrying in our regions. The main results achieved can be summarized as: A male predominance with a sex ratio of 1.38; A peak incidence between 65 and 70years with an average age of 68 years; they were without full-time professional activity for most and they were also without health insurance; In all cases it is type 2 diabetes. In $39 \%$ of cases, the ketoacidosis of the elderly over 65 years was inaugural of diabetes. Triggers were dominated by infection in $51.6 \%$ of cases with malaria as main infection. All patients had blood glucose higher than $3 \mathrm{~g} / 1$ with average blood glucose of $3.8 \mathrm{~g} / \mathrm{l}$. The average length of hospital stay was 6 days. $84 \%$ of patients had a high probability of depression. $85.4 \%$ of patients had a strong suspicion of cognitive impairment. Therapeutic measures of care for diabetic ketoacidosis among the aged should be rigorous to prevent the onset of complications.

\section{Acknowledgements}

None.

\section{Conflict of interest}

Author declares that there is no conflict of interest.

\section{References}

1. World Health Organization. Definition, diagnosis and complications of diabetes mellitus and its complications. Part1: diagnosis and classification of diabetes mellitus. Geneva: WHO. 1999.

2. Alberti KG, Zimmet PZ. Definition, diagnosis, and classification of diabetes mellitus and its complications. Part1: diagnosis and classification of diabetes mellitus provisional report of a WHO consulation. Diabet Med. 1998;15(7):539-553.

3. Gning SB, Thiam M, Fall F, et al. Diabète sucré en Afrique subsaharienne: aspects épidémiologiques, difficultés de prise en charge. Medecine tropicale. 2007;67(1):607-611.

4. Fati N. Evaluation gérontologique: pour quiet comment? Rev Prat Méd Gen. 2010;23(817):168-169.

5. Abodo JR, P Koffi Dago, Kouassi. Diabetologic and geriatric characteristics of the elderly diabétic patient hospitalized in service of endocrinology and diabetology of the university hospital of yopougon. Abidjan Rev International de sciences med. 2012;14(2):2-11.

6. Alix M. Diabète chez le sujet âgé. Presse Med. 2000;2:2150-2155.

7. Bourdel, Marchasson I. Régulation de la glycémie jusqu'ouet quand chez le sujet âgé? Journ annv Hotel Dieu. 2005. p. 155-156.

8. Belmin J, Chassagne P, Friocort P, et al. Gériatrie pour le praticien. France: Editions Masson; 2009. 110p.

9. Remington R, Hultman T. Hypodermoglysis to treat dehydration a review of the evidence. J Am Geriatr Soc. 2007;55(12):2051-2055. 
10. Capet C, Jego AK, Drvenel. Hypernatrémie du sujet agé. Rev Gen Gerontol. 1999;56:18-21.

11. Grimaldi A. L'acidocétose diabétique. In: Les diabèteset les hypoglycémies. France, Paris: JB Baillère; 1985. p. 86-100.

12. Intergroupe diabétogériatrie ALFEDIAM/SFGG. Guide pour la prise en charge du diabétique âgé. Med Mal Metab hors-série1, 2. 2008.

13. Dorner M, Kuntzmann F, Brogard JM. Evolution, complications spontanées et induites, pronostic et traitement des accidents acidocétosiques et du coma diabétique. Erreurs à ne pas commettre. Rev Prat . 1970;20(31):5021-5037.

14. Chiasson JL, Aris Jilwan N, Belanger R, et al. Diagnosis and treatment of diabetic ketoacidosis and the hyperglycaemic hyperosmolar state. CMAJ. 2003;168(7):859-866.

15. Sinclair A J. European Diabetes Working Party for Older People, Clinical Guidelines for type 2 Diabetes Mellitus. 2004

16. Lokrou A. Traitement du coma acidocétosique: aspects actuels. Sem Hop Paris. 1992;68(6):154-160.

17. Vischer UM, Perrenoud L, Genet C, et al. The high prevalence of malnutrition in elderly diabetic patients: implications for anti-diabetic drug treatments. Diabet Med. 2010;27(8):918-924.

18. Anderson RJ, Freeland KE, Clouse RE, et al. The prevalence of comorbid depression in adultes with diabetes: a meta-analysis. Diabetes care. 2001;24(6):1069-1078.

19. Bourdel Marchasson I, Dubroca B, Manciet G, et al. Prevalence of diabetes and effect on quality of life in older french living in community: the PAQUID Epidemiological Survery. J Am Geriatr Soc. 1997;45(3):295-301.
20. Black SA. Increased breadline burden associated with comorbid depression in older diabetic Mexican Americans. Results from the Hispanic Established Population for the Epidemiologic Study of the Elderly survey. Diabetes care. 1999;22(1):56-64.

21. Rosenthal MJ, Pajardo M, Gilmore S, et al. Hospitalization and mortality of diabetes in older adults. A 3year prospective study. Diabetes care. 1998;21(2):231-235.

22. Lustman PJ, Anderson RJ, Freeland KE, et al. Depression and poor glycemic control. A meta-analytic review of the literature. Diabetes care. 2000;23(7):934-942.

23. Meneilly GS, Tessier D. Diabetes in elderly. Diabetes Med. 1995;12:949-960.

24. Kumari M, Brunner E, Fuhrier R. Mini review: mechanisms by which the metabolic syndrome and diabetes impair memory. Gerontol Biol Sci. 2000;55A:B228-B232.

25. Gregg EW, Yaffe K, Cavley JA, et al. Is diabetes associated with cognitive impairment and cognitive decline among older women? Study of Osteoporotic Fractures Research Group. Arch Intern Med. 2000;160(2):174-180.

26. Gradman TJ, Laws A, Thompson W, et al. Verbal learning and/ or memory improves with glycemic control in older subjects with non-insulin-dependent diabetes mellitus. $J$ Am Geriatr Soc. 1993;41(12):1305-1312.

27. Meneilly GJ, Chevng E, Tessier D, et al. The affect of improved glycemic control on cognitive functions in the elderly patient with diabetes. J Gerontol. 1993;48(4):M117-M121. 